J.-T. LU

KODAI MATH. J.

17 (1994), 290-298

\title{
HYPERSURFACES OF A SPHERE WITH 3-TYPE QUADRIC REPRESENTATION
}

\author{
BY JI-TAN LU
}

\begin{abstract}
We study hypersurfaces of a sphere with 3-type quadric representation. Two theorems are obtained, and some eigenvalue inequalities are proved.
\end{abstract}

\section{Introduction}

Let $\Phi: M^{n} \rightarrow E^{m}$ be an isometric immersion of an $n$-dimensional compact Riemannian manifold into the Euclidean space, $\Delta$ and $\operatorname{spec}\left(M^{n}\right)=\left\{0<\lambda_{1}<\lambda_{2}<\cdots\right.$ $\nearrow+\infty\}$ be the Laplacian and the spectrum of $M^{n}$, respectively. Then we have the decomposition $\Phi=\sum_{u \geq 0} \Phi_{u}, u \in N$, where $\Phi_{u}: M^{n} \rightarrow E^{m}$ is a differentiable mapping such that $\Delta \Phi_{u}=\lambda_{u} \Phi_{u}$, moreover $\Phi_{0}$ is a constant mapping (it is the center of mass of $M^{n}$ ). $M^{n}$ is said to be of finite type if the decomposition consists of only a finite number of non-zero terms, and of $k$-type if there are exactly $k$ non-zero $\Phi_{u}$ 's $\left(\Phi_{u_{1}}, \cdots, \Phi_{u_{k}}\right)$ in the decomposition. In the latter case, we also call the immersion $\Phi$ to be of $k$-type.

Finite type submanifolds of a hypersphere $S^{m-1} \subset R^{m}$ have been studied by many authors. For example, see [5], [2], [9], [3]. In [5] mass-symmetric 2type hypersurfaces of $S^{m-1}$ were characterized. In [2] it was proved that a compact 2-type hypersurface of $S^{m-1}$ is mass-symmetric if and only if it has constant mean curvature. In [9] Nagatomo showed that many 2-type hypersurfaces of a hypersphere are mass-symmetric and that there is no compact hypersurface of constant mean curvature in a hypersphere which is of 3-type. In particular, Barros and Garay [3] proved that the Riemannian product of two plane circles of different and stuitable radii is the only 2-type surfaces in $S^{3} \subset R^{4}$.

On the other hand, let $\Psi: M^{n} \rightarrow S^{n+p}(1)$ be a minimal isometric immersion of an $n$-dimensional compact Riemannian manifold into the unit sphere, $S M(n+$ $p+1)=\left\{P \in g l(n+p+1, R) \mid P^{t}=P\right\}$, and $f: S^{n+p}(1) \rightarrow S M(n+p+1)$ be the order 2 immersion of $S^{n+p}(1)$. We consider the associated isometric immersion $\Phi=$ $f \circ \Psi: M^{n} \rightarrow S M(n+p+1)$, which is called the quadric representation of $M^{n}$. In [8], Ros characterized minimal submanifolds in $S^{n+p}(1)$ with 2-type quadric representation. Later, $\mathrm{Lu}$ [7] proved that the Clifford torus $M_{m, m}$ are the only

Received September 28, 1993. 
full compact minimal hypersurfaces in $S^{2 m+1}(1)$ with 2-type representation and that the Veronese surfaces in $S^{4}$ are the only full compact minimal surfaces in the unit sphere for which $\Phi$ is of 2-type. In this paper we study hypersurfaces of a sphere with 3-type quadric representation. Our main results are

THEOREM 1. Let $M^{n}$ be a compact minımal hypersurface of a sphere with 3-type quadric representation. Then the length of the second fundamental form of $M^{n}$ in the sphere must be constant.

On the basis of Theorem 1, we further prove

THEOREM 2. There does not exist compact minimal surface in $S^{3}(1)$ with 3-type quadric representation.

Theorem 2 is not valid for any dimensional compact minimal hypersurfaces in a sphere. For example, minimal Cartan hypersurface $S O(3) / Z_{2} \times Z_{2}$ in $S^{4}$ is showed to just have 3-type quadric representation.

Finally we also give some eigenvalue inequalities. The author wishes to thank professor W.H. Chen for many valuable comments and suggestions.

\section{Preliminaries}

Let $S^{n+p}(1)$ be an Euclidean sphere with radius 1 and $S M(n+p+1)=\{P \in$ $\left.g l(n+p+1, R) \mid P^{t}=P\right\}$ be the space of the real symmetric matrices of order $n+p+1$. We define on $S M(n+p+1)$ the metric $\langle P, Q\rangle=(1 / 2) \operatorname{tr} P Q$, for arbitrary $P, Q$ in $S M(n+p+1)$. We consider the mapping $f: S^{n+p}(1) \rightarrow S M(n+p+1)$ given by $f(x)=x x^{t}$, where $x$ is the position column vector of $S^{n+p}(1)$ in $R^{n+p+1}$, and $x^{t}$ is the transpose of $x$. Then $f$ is the order 2 immersion of the sphere, and the mass center of $f\left(S^{n+p}(1)\right)$ is $I /(n+p+1)$, where $I$ is the identity matrix in $S M(n+p+1)$. We identify $x$ with $f(x)$. Then the normal space for the immersion $f$ at $x$ of $S^{n+p}(1)$ is given by

$$
T_{x}^{\perp}\left(S^{n+p}(1)\right)=\{Q \in S M(n+p+1) \mid Q x=\lambda x \text {, for some real } \lambda\} .
$$

We denote by $\bar{\nabla}$, $\tilde{\nabla}$ the Riemannian connection on $S M(n+p+1)$ and $S^{n+p}(1)$, respectively, and by $\tilde{\sigma}, \tilde{A}$ and $\widetilde{H}$ the second fundamental form, the Weingarten endomorphism and the mean curvature vector of immersion $f$, respectively, the normal connection of $f$ is denoted by $\widetilde{D}$. Then we have the following formulas

$$
\begin{aligned}
& \tilde{D}_{\tilde{\sigma}}=0, \\
& \tilde{H}_{x}=\frac{2}{n+p}(I-(n+p+1) x), \\
& \langle\tilde{\sigma}(X, Y), \tilde{\sigma}(V, W)\rangle=2\langle X, Y\rangle\langle V, W\rangle+\langle X, V\rangle\langle Y, W\rangle+\langle X, W\rangle\langle Y, V\rangle, \\
& \tilde{A}_{\tilde{\sigma}(X, Y)} V=2\langle X, Y\rangle V+\langle X, V\rangle Y+\langle Y, V\rangle X,
\end{aligned}
$$




$$
\langle x, \tilde{\sigma}(X, Y)\rangle=-\langle X, Y\rangle,
$$

$$
\langle I, \tilde{\sigma}(X, Y)\rangle=0 \text {, }
$$

where $X, Y, V, W$ are vector fields tangent to $S^{n+p}(1)$.

\section{Compact minimal hypersurfaces in the sphere}

Let $\Psi: M^{n} \rightarrow S^{n+1}(1)$ be a minimal isometric immersion of a hypersuface in $S^{n+1}(1)$. Let $e_{1}, \cdots, e_{n}, N$ be a local field of orthonormal frames of $S^{n+1}(1)$, such that restricted to $M^{n}, e_{1}, \cdots, e_{n}$ are tangent to $M^{n}$. We denote by $\nabla, D$ the Riemannian connection of $M^{n}$ and the normal connection of $\Psi$, and by $\sigma$, $A, H$ the second fundamental form, the Weingarten endomorphism and the mean curvature vector of $\Psi$, respectively. Considering the associated isometric immerison $\Phi=f \circ \Psi: M^{n} \rightarrow S M(n+2)$, we have the following formulas (see [8])

$$
\begin{gathered}
\Delta \Phi=-\sum_{\imath=1}^{n} \tilde{\sigma}\left(e_{\imath}, e_{\imath}\right), \\
\Delta^{2} \Phi=2(n+1) \Delta \Phi-2 \sum_{\imath, j} \tilde{\sigma}\left(A_{\sigma\left(e_{i}, e_{j}\right)} e_{\imath}, e_{j}\right)+2 \sum_{\imath, j} \tilde{\sigma}\left(\sigma\left(e_{\imath}, e_{\jmath}\right), \sigma\left(e_{\imath}, e_{\jmath}\right)\right) .
\end{gathered}
$$

We denote by $S$ the square of the length of $\sigma$, then from (2.2) we have

$$
\Delta^{2} \Phi=2(n+1) \Delta \Phi+2 S \tilde{\boldsymbol{\sigma}}(N, N)-2 \sum_{\imath=1}^{n} \tilde{\boldsymbol{\sigma}}\left(A e_{\imath}, A e_{\imath}\right) .
$$

Let $\left(\nabla_{X} A\right) Y=\nabla_{X}(A Y)-A\left(\nabla_{X} Y\right)$, for arbitrary vector feilds $X, Y$ tangent to $M^{n}$, and $\Delta A=-\sum_{k=1}^{n} \nabla_{e_{k}}\left(\nabla_{e_{k}} A\right)+\sum_{k=1}^{n} \nabla_{\nabla_{e_{k} e_{k}}} A$. We will prove the following Lemmas.

LEMMA 1. Let $\Psi: M^{n} \rightarrow S^{n+1}(1)$ be a minimal isometric immersion of a hypersurface into the sphere, $\Phi=f \circ \Psi$. Then

$$
\begin{aligned}
\Delta^{3} \Phi= & \left(2 \Delta S+4 S^{2}+4\left|A^{2}\right|^{2}\right) \tilde{\sigma}(N, N) \\
& -4 \operatorname{grad} S-8\left(\operatorname{tr} A^{3}\right) N+2(n+1) \Delta^{2} \Phi \\
& +12 \tilde{\sigma}(N, A \operatorname{grad} S)+\frac{8}{3} \tilde{\sigma}\left(N, \operatorname{grad}\left(\operatorname{tr} A^{3}\right)\right) \\
& -4(S+1) \sum_{k=1}^{n} \tilde{\sigma}\left(A e_{k}, A e_{k}\right)-4 \sum_{k=1}^{n} \tilde{\sigma}\left(A^{2} e_{k}, A^{2} e_{k}\right) \\
& -4 \sum_{k=1}^{n} \tilde{\sigma}\left((\Delta A)\left(e_{k}\right), A e_{k}\right)+4 \sum_{i, k=1}^{n} \tilde{\sigma}\left(\left(\nabla_{e_{k}} A\right)\left(e_{\imath}\right),\left(\nabla_{e_{k}} A\right)\left(e_{2}\right)\right) .
\end{aligned}
$$

Proof. At first we compute the differential of $\Delta^{2} \Phi$. From (2.3) we obtain 


$$
\begin{aligned}
\left(\Delta^{2} \Phi\right)_{*}\left(e_{k}\right)= & 2(n+1)(\Delta \Phi)_{*}\left(e_{k}\right)+2 e_{k}(S) \tilde{\sigma}(N, N) \\
& +2 S \bar{\nabla}_{e_{k}} \tilde{\sigma}(N, N)-2 \sum_{\imath=1}^{n} \bar{\nabla}_{e_{k}} \tilde{\sigma}\left(A e_{\imath}, A e_{\imath}\right) .
\end{aligned}
$$

We compute the last two terms respectively.

$$
\bar{\nabla}_{e_{k}} \tilde{\sigma}(N, N)=-\tilde{A}_{\tilde{\sigma}(N, N)} e_{k}+2 \tilde{\sigma}\left(\tilde{\nabla}_{e_{k}} N, N\right)=-2 e_{k}-2 \tilde{\sigma}\left(A e_{k}, N\right),
$$

where we have used (1.5), (1.2) and $D_{e_{k}} N=0$. By the same way we have

$$
\begin{aligned}
\sum_{\imath=1}^{n} \bar{\nabla}_{e_{k}} \tilde{\sigma}\left(A e_{\imath}, A e_{\imath}\right)= & -\sum_{\imath=1}^{n} \tilde{A}_{\tilde{\sigma}\left(A e_{i}, A e_{i}\right)} e_{k}+\sum_{\imath=1}^{n} \tilde{D}_{e_{k}} \tilde{\sigma}\left(A e_{\imath}, A e_{\imath}\right) \\
= & -2 S e_{k}-2 \sum_{\imath=1}^{n}\left\langle A e_{\imath}, e_{k}\right\rangle A e_{\imath} \\
& +2 \sum_{\imath=1}^{n} \tilde{\sigma}\left(\sigma\left(e_{k}, A e_{\imath}\right), A e_{\imath}\right)+2 \sum_{\imath=1}^{n} \tilde{\sigma}\left(\nabla_{e_{k}}\left(A e_{\imath}\right), A e_{\imath}\right) .
\end{aligned}
$$

Hence, from (2.5), (2.6) and (2.7) we have

$$
\begin{aligned}
\left(\Delta^{2} \Phi\right)_{*}\left(e_{k}\right)= & -4 S \tilde{\sigma}\left(A e_{k}, N\right)+4 A^{2} e_{k} \\
& +2(n+1)(\Delta \Phi)_{*}\left(e_{k}\right)+2 e_{k}(S) \tilde{\sigma}(N, N) \\
& -4 \sum_{\imath=1}^{n} \tilde{\sigma}\left(\sigma\left(e_{k}, A e_{\imath}\right), A e_{\imath}\right)-4 \sum_{\imath=1}^{n} \tilde{\sigma}\left(\nabla_{e_{k}}\left(A e_{\imath}\right), A e_{\imath}\right) .
\end{aligned}
$$

Let $x$ be an arbitrary point in $M^{n}$, we may assume that $\nabla_{e_{\jmath}} e_{\imath}=0$ at $x$. We compute $\Delta^{3} \Phi$ at $x$ as follows

$$
\begin{aligned}
\Delta^{3} \Phi(x)= & -\sum_{\imath=1}^{n} \bar{\nabla} e_{k}\left(\Delta^{2} \Phi\right)_{*}\left(e_{k}\right) \\
= & 2(n+1) \Delta^{2} \Phi(x)+2 \Delta(S) \tilde{\sigma}(N, N) \\
& +4 S \sum_{k=1}^{n} \bar{\nabla}_{e_{k}} \tilde{\sigma}\left(A e_{k}, N\right)-4 \sum_{k=1}^{n} \bar{\nabla}_{e_{k}}\left(A^{2} e_{k}\right) \\
& +4 \sum_{k=1}^{n} e_{k}(S) \tilde{\sigma}\left(A e_{k}, N\right)-2 \sum_{k=1}^{n} e_{k}(S) \bar{\nabla}_{e_{k}} \tilde{\sigma}(N, N) \\
& +4 \sum_{k, \imath=1}^{n} \bar{\nabla}_{e_{k}} \tilde{\sigma}\left(\sigma\left(e_{k}, A e_{\imath}\right), A e_{\imath}\right)+4 \sum_{k=1}^{n} \bar{\nabla}_{e_{k}} \tilde{\sigma}\left(\nabla_{e_{k}}\left(A e_{\imath}\right), A e_{\imath}\right) .
\end{aligned}
$$

It is obvious that

$$
\sum_{k=1}^{n} e_{k}(S) \tilde{\sigma}\left(A e_{k}, N\right)=\tilde{\sigma}(A \operatorname{grad} S, N) \text {. }
$$

By a direct computation, using Codazzi equation, $H=0$ and (1.2), (1.5) we obtain the following relations 


$$
\begin{aligned}
& \sum_{k=1}^{n} \bar{\nabla}_{e_{k}} \tilde{\sigma}\left(A e_{k}, N\right)=S \tilde{\sigma}(N, N)-\sum_{k=1}^{n} \tilde{\sigma}\left(A e_{k}, A e_{k}\right), \\
& \sum_{k=1}^{n} \bar{\nabla}_{e_{k}}\left(A^{2} e_{k}\right)=\sum_{k=1}^{n} \tilde{\sigma}\left(A e_{k}, A e_{k}\right)+\left(\operatorname{tr} A^{3}\right) N+\frac{1}{2} \operatorname{grad} S, \\
& \sum_{k=1}^{n} \bar{\nabla}_{e_{k}} \tilde{\sigma}\left(\nabla_{e_{k}}\left(A e_{\imath}\right), A e_{\imath}\right)=\frac{1}{3} \tilde{\sigma}\left(N, \operatorname{grad}\left(\operatorname{tr} A^{3}\right)\right)-\sum_{k=1}^{n} \tilde{\sigma}\left((\Delta A)\left(e_{\imath}\right), A e_{\imath}\right) \\
& \quad-\frac{3}{2} \operatorname{grad} S+\frac{1}{2} \tilde{\sigma}(N, A \operatorname{grad} S)+\sum_{k, \imath=1}^{n} \tilde{\sigma}\left(\left(\nabla_{e_{k}} A\right)\left(e_{\imath}\right),\left(\nabla_{e_{k}} A\right)\left(e_{\imath}\right)\right), \\
& \sum_{k=1}^{n} \bar{\nabla}_{e_{k}} \tilde{\sigma}\left(\sigma\left(e_{k}, A e_{\imath}\right), A e_{\imath}\right)=\frac{1}{2} \tilde{\sigma}(N, A \operatorname{grad} S) \\
& \quad+\frac{1}{3} \tilde{\sigma}\left(N, \operatorname{grad}\left(\operatorname{tr} A^{3}\right)\right)-\sum_{k=1}^{n} \tilde{\sigma}\left(A^{2} e_{k}, A^{2} e_{k}\right)-\operatorname{tr}\left(A^{3}\right) N+\left|A^{2}\right|^{2} \tilde{\sigma}(N, N) .
\end{aligned}
$$

From (2.6), (2.9) and the above relations we have (2.4).

LEMMA 2. Let $\Psi: M^{n} \rightarrow S^{n+1}(1)$ be a minimal isometric immersion of a hypersurface in the sphere, $\Phi=f \circ \Psi$. Then we have the following relations

$$
\begin{aligned}
& \langle\Phi, \Phi\rangle=\frac{1}{2}, \\
& \langle\Phi, \Delta \Phi\rangle=n, \\
& \left\langle\Phi, \Delta^{2} \Phi\right\rangle=2 n(n+1), \\
& \left\langle\Phi, \Delta^{3} \Phi\right\rangle=4 n(n+1)^{2}+S, \\
& \left\langle\Delta \Phi, \Delta^{2} \Phi\right\rangle=4 n(n+1)^{2}+S .
\end{aligned}
$$

Proof. The above relations can be obtainted by a long but direct computation using (2.1), (2.2), (2.4), (1.4) and (1.6).

Note. In fact, (2.10), (2.11), (2.12), (2.14) hold for any co-dimention $p$. (see [8], Lemma 2.2).

\section{Proof of the Theorems 1 and 2}

Proof of Theorem 1. Let $\Psi: M^{n} \rightarrow S^{n+1}(1)$ be a minimal isometric immersion, $\Phi=f \circ \Psi$, if $\Phi$ is of 3 -type $\left(\left\{u_{1}, u_{2}, u_{3}\right\}\right)$. Then we have

and

$$
\Phi=\Phi_{0}+\Phi_{u_{1}}+\Phi_{u_{2}}+\Phi_{u_{3}}
$$

$$
\begin{aligned}
& \Delta \Phi=\lambda_{u_{1}} \Phi_{u_{1}}+\lambda_{u_{2}} \Phi_{u_{2}}+\lambda_{u_{3}} \Phi_{u_{3}}, \\
& \Delta^{2} \Phi=\lambda_{u_{1}}^{2} \Phi_{u_{1}}+\lambda_{u_{2}}^{2} \Phi_{u_{2}}+\lambda_{u_{3}}^{2} \Phi_{u_{3}},
\end{aligned}
$$


Hence

$$
\Delta^{3} \Phi=\lambda_{u_{1}}^{3} \Phi_{u_{1}}+\lambda_{u_{2}}^{3} \Phi_{u_{2}}+\lambda_{u_{3}}^{3} \Phi_{u_{3}} .
$$

$$
\Delta^{3} \Phi=a \Delta^{2} \Phi+b \Delta \Phi+c \Phi-c \Phi_{0}
$$

where

$$
a=\sum_{i=1}^{3} \lambda_{u_{i}}, b=-\sum_{1 \leqq i<j \leqq 3} \lambda_{u_{i}} \lambda_{u_{j}}, c=\prod_{\imath=1}^{3} \lambda_{u_{i}} .
$$

From (1.1), (2.1) and (2.2) we know that $I, \Phi, \Delta \Phi$ and $\Delta^{2} \Phi$ are all normal to $S^{n+1}(1)$. Hence, for any vector field $X$ tangent to $M^{n}$, we use (2.4) and (3.1) to obtain

$$
\left\langle\Delta^{3} \Phi, X\right\rangle=-4\langle\operatorname{grad} S, X\rangle=-c\left\langle X, \Phi_{0}\right\rangle,
$$

but

and

$$
X\left\langle\Phi, \Phi_{0}\right\rangle=\left\langle X, \Phi_{0}\right\rangle,
$$

Therefore

$$
\langle\operatorname{grad} S, X\rangle=X(S) \text {. }
$$

This means

$$
X\left(4 S-c\left\langle\Phi, \Phi_{0}\right\rangle\right)=0 .
$$

$$
4 S-c\left\langle\Phi, \Phi_{0}\right\rangle=\text { constant } .
$$

On the other hand, by using (2.10), (2.11), (2.12) and (3.1) we have

$$
\left\langle\Delta^{3} \Phi, \Phi\right\rangle=2 n(n+1) a+n b+\frac{1}{2} c-c\left\langle\Phi_{0}, \Phi\right\rangle .
$$

Combining with (2.14), we have

$$
S+c\left\langle\Phi, \Phi_{0}\right\rangle=2 n(n+1) a+n b+\frac{1}{2} c-4(n+1)^{2} n
$$

Hence, from (3.3) and (3.2) we obtain $S=$ constant, and $\left\langle\Phi, \Phi_{0}\right\rangle=$ constant. This finishes the proof of Theorem 1.

Proof of Theorem 2. When $n=2$, i.e, $M^{2}$ is a compact minimal surface in $S^{3}(1)$. For the Gauss curvature $K$ of $M^{2}$, we have $K=1-(1 / 2) S$. If $\Phi$ is of 3-type, from Theorem 1, we know that $K$ is constant. But Bryant [1] had proved that there is no minimal surface of constant negative Guassian curvature in $S^{n}$, so $K \geqq 0$. If $K=0$, then $S=2$. From the well-known result of Chern and others [6], we know that $M^{2}$ must be the Clifford torus $M_{1,1}$. But we know that for $M_{1,1} \Phi$ is of 2-type. This is a contradiction in consideration of $\Phi$ being of 3-type. If $K>0$, Calabi [4] told us $K$ must be 1 , thus, $S=0$. This means that $M^{2}$ is the geodesic sphere and therefore $\Phi$ is of 1 -type. This is also a contradiction to that $\Phi$ is of 3 -type. Theorem 2 is thereby proved. 


\section{Eigenvalue inequalities}

Let $\Psi: M^{n} \rightarrow S^{n+p}(1)$ be a minimal isometric immersion of a compact $n$ dimentional Riemannian manifold into the sphere. Then $\Phi=f \circ \Psi$ is an isometric immersion of $M^{n}$ into $S M(n+p+1, R)$. Let $\Phi=\sum_{u \geqq 0} \Phi_{u}$ be the spectral decomposition. Then we have

$$
\int_{M n}\left\langle\Phi_{u}, \Phi_{v}\right\rangle * 1=0 \quad \text { for all } u, v \in N, u \neq v,
$$

where $* 1$ is the volume element of $M^{n}$.

We put

$$
\int_{M n}\left\langle\Phi_{u}, \Phi_{u}\right\rangle * 1=a_{u} \quad \text { for all } \quad u \in N
$$

and

$$
\Omega_{k}=\int_{M}\langle\Delta \Phi, \Phi\rangle * 1-\lambda_{k} \int_{M}\left\langle\Phi-\Phi_{0}, \Phi\right\rangle * 1
$$

then

$$
\int_{M n}\left\langle\Delta^{3} \Phi, \Phi\right\rangle * 1=\sum_{u \geqq 1} \lambda_{u}^{3} a_{u} .
$$

From the above relations, we obtain

$$
\Omega_{1}=\sum_{u>1}\left(\lambda_{u}-\lambda_{1}\right) a_{u} \geqq 0
$$

the equality in (4.3) holds if and only if $\Phi$ is of order 1.

THEOREM 3. Let $\Psi: M^{n} \rightarrow S^{n+p}(1)$ be an minimal isometric immersion, and $\Phi=f \circ \Psi . \quad$ Then

$$
\lambda_{1} \leqq \frac{n}{(1 / 2)-\left|\Phi_{0}\right|^{2}},
$$

the equality holds if and only if $\Phi$ is of order 1 .

The theorem is obtained from (4.3), (2.10) and (2.11).

THEOREM 4. Let $\Psi: M^{n} \rightarrow S^{n+p}(1)$ be a minimal isometric immersion, and $\Phi=f \circ \Psi$. If $\Phi$ is of 3-type, then we have

$$
\begin{aligned}
& 2 n(n+1) \sum_{\imath=1}^{3} \lambda_{u_{\imath}}-n \sum_{\imath<j} \lambda_{u_{i}} \lambda_{u_{\jmath}} \\
& \quad+\frac{n+p}{2(n+p+1)} \prod_{\imath=1}^{3} \lambda_{u_{\imath}}-4 n(n+1)^{2} \geqq \frac{\int_{M n} S^{* 1}}{\operatorname{Vol}\left(M^{n}\right)},
\end{aligned}
$$


the equality holds of and only if the centres of $M^{n}$ and $S^{n+p}(1)$ in $S M(n+p+1)$ are the same.

Proof. If $\Phi$ is of 3-type, we have

$$
\Delta^{3} \Phi=\left(\sum_{\imath=1}^{3} \lambda_{u_{\imath}}\right) \Delta^{2} \Phi-\left(\sum_{\imath<j} \lambda_{u_{i}} \lambda_{u_{\jmath}}\right) \Delta \Phi+\left(\prod_{\imath=1}^{3} \lambda_{u_{\imath}}\right)\left(\Phi-\Phi_{0}\right) .
$$

Then

$$
\begin{aligned}
& \int_{M n}\left\langle\Delta^{3} \Phi, \Phi\right\rangle * 1=\left(\sum_{\imath=1}^{3} \lambda_{u_{\imath}}\right) \int_{M}\left\langle\Delta^{2} \Phi, \Phi\right\rangle * 1 \\
& -\left(\sum_{\imath<j} \lambda_{u_{i}} \lambda_{u_{\jmath}}\right) \int_{M}\langle\Delta \Phi, \Phi\rangle * 1+\left(\prod_{\imath=1}^{3} \lambda_{u_{\imath}}\right)\left(\int_{M}\langle\Phi, \Phi\rangle * 1-\int_{M}\left\langle\Phi, \Phi_{0}\right\rangle * 1\right) .
\end{aligned}
$$

Using (2.14), (2.12), (2.11), (2.10) and (4.2), we get

$$
\begin{aligned}
2 n(n+1)\left(\sum_{\imath=1}^{3} \lambda_{u_{\imath}}\right)-n\left(\sum_{\imath<j} \lambda_{u_{i}} \lambda_{u_{\jmath}}\right) \\
+\left(\frac{1}{2}-\left|\Phi_{0}\right|^{2}\right)\left(\prod_{\imath=1}^{3} \lambda_{u_{\imath}}\right)-4 n(n+1)^{2}=\frac{\int_{M n} S^{* 1}}{\operatorname{Vol}\left(M^{n}\right)},
\end{aligned}
$$

where $\operatorname{Vol}\left(M^{n}\right)$ is the volume of $M^{n}$.

We recall that $\Phi_{0}$ is a constant mapping and $\Phi_{0}=\int_{M n} \Phi^{*} 1 / \operatorname{Vol}\left(M^{n}\right)$. So,

$$
\operatorname{tr} \Phi_{0}=\operatorname{tr}\left(\frac{\int_{M n} \Phi^{* 1}}{\operatorname{Vol}\left(M^{n}\right)}\right)=\frac{\int_{M n} \operatorname{tr} \Phi^{* 1}}{\operatorname{Vol}\left(M^{n}\right)}=1,
$$

where we use the fact that $\operatorname{tr} \Phi=1$. and

$$
\left|\Phi_{0}\right|^{2}=\frac{1}{2} \sum_{i=1}^{n+p+1} \mu_{\imath}^{2} \geqq \frac{1}{2(n+p+1)}\left(\sum_{i=1}^{n+p+1} \mu_{\imath}\right)^{2}=\frac{1}{2(n+p+1)},
$$

the equality holds if and only if $\mu_{1}=\cdots=\mu_{n+p+1}$. This means $\Phi_{0}=1 /(n+p+1)$. Combining (4.9) and (4.7) we have (4.4).

\section{REFERENCES}

[1] R. L. BRyAnt, Minimal surfaces of constant curvature in $S^{n}$, Trans. Amer. Math. Soc., 290 (1985), 259-271.

[2] M. Barros, B. Y. Chen and O.J. Garay, Spherical finite type hypersurfaces, Algebras Groups Geom., 4 (1987), 58-72.

[3] M. Barros and O.J. Garay, 2-type surfaces in $S^{3}$, Geom. Dedicata, 14 (1987), 329-336.

[4] E. CALABI, Minimal immersions of surfaces in Euclidean spheres, J. Differential Geom., 1 (1967), 111-125. 
[5] B. Y. Chen, Total Mean Curvature and Submanifolds of Finite Type, World Scientific, 1984.

[6] S.S. Chern, M. do Carmo and S. Kobayashi, Minimal submanifolds of a sphere with second fundamental form of constant length, Shing-Shen Chern Selected Papers, Springer-verlag, 1978, 393-409.

[7] J.T. Lu, Spectral geometry of minimal submanifolds in $S^{n}(1)$, J. Tsinghua Univ., 33 (1993) S4, 108-113.

[8] A. Ros, Eigenvalue inequalities for minimal submanifolds and P-manifolds, Math. Z., 187 (1984), 393-404.

[9] Y. Nagatomo, Finite type hypersurfaces of a sphere, Tokyo J. Math., 14 (1991), 85-92.

[10] I. Dimitric, Quadric representation of a submanifold, Proc. Amer. Math. Soc., 114 (1992), 201-210.

Department of Applied Mathematics

TsinghuA University (100084)

Beijing, China 